\title{
DIABETES MELLITUS TIPO II: ASSISTÊNCIA À SAÚDE EM RELAÇÃO AO GÊNERO
}

\author{
Tatiana Longo \\ Graduada em Enfermagem/UNIPAR \\ tatianalongo@bol.com
}

Edinalva Madalena de Almeida Mota

Mestre em Promoção da Saúde/UNICESUMAR; edinalva.carraro@bol.com.br

\section{RESUMO}

O Diabetes mellitus é uma doença que vem crescendo em amplo espectro, acarretando em sérias complicações como perda da visão, disfunções renais, amputações entre outras complicações, tanto em mulheres como em homens. É preciso conhecer a epidemiologia da doença para delimitar a população mais acometida e traçar metas elaborando intervenções mediante a necessidade de adaptação de cada indivíduo respeitando as peculiaridades de cada sexo. Objetivou-se com a presente pesquisa conhecer a prevalência do Diabetes mellitus segundo sexo e a relação com a busca pelos serviços de saúde por homens e mulheres a fim de sensibilizar os profissionais de saúde o quanto é importante estabelecer programas de atenção para o portador da doença. A prevalência na maior parte dos estudos analisados se constitui do sexo feminino, estes dados trazem consigo reflexões a cerca dos motivos que fazem da mulher a mais acometida pelo Diabetes mellitus. Vários fatores podem ser relacionados como o papel da mulher atrelada a ser cuidadora, a instabilidade hormonal no climatério, o da mulher buscar mais os serviços se saúde, logo é diagnosticada precocemente, além dos programas de saúde estarem mais voltadas a saúde da mulher o que facilita o acesso. É preciso despertar nos profissionais de saúde a necessidade de se trabalhar questões de gênero em saúde abrangendo as doenças crônicas não transmissíveis para que os pacientes tanto homem como mulher alcancem o melhor estado de saúde com uma assistência adequada e preventiva respeitando essas diferenças biológicas.

Palavras-chave: Diabetes mellitus; Diabetes mellitus Tipo II; Assistência em saúde. Educação e Saúde; Enfermagem.

\begin{abstract}
Diabetes mellitus is a disease that is growing in a wide range, resulting in serious complications such as vision loss, kidney failure, amputations and other complications, both in women as in men. You need to know the epidemiology of Diabetes mellitus to define the population most affected and set goals by developing interventions need to adapt to each individual respecting the peculiarities of each sex. The objective of this research to determine the prevalence of diabetes mellitus by sex and relationship with the search for health services by men and women with disease in order to educate health professionals how important it is to establish programs to both the health of man as the woman with Diabetes mellitus. The prevalence in most studies is examined if female, these data bring about thoughts of the reasons that make women more affected by diabetes. Several factors may be related to the role of women linked to caregiver, hormonal instability in menopause, the woman is seeking more health care services, so it is diagnosed early, and health programs are more focused on women's health which facilitates access. We must awaken in health professionals need to work with gender issues in health covering chronic diseases so that both male and female patients reach the best state of health with adequate assistance and preventive biological respecting these differences.
\end{abstract}

Keywords: Diabetes mellitus; Diabetes mellitus Type II; Health care. Education and Health; Nursing.

Persp. online: biol. \& saúde, Campos dos Goytacazes, 16 (5). P.1-10, 2015

seer.perspectivasonline.com.br 


\section{INTRODUÇÃO}

O processo de envelhecimento da população propicia a transição do perfil epidemiológico, uma das consequências desta mudança é o aumento progressivo da incidência das doenças crônicas não transmissíveis, principalmente do Diabetes mellitus como uma das mais relevantes (COSTA et al., 2006).

De acordo com Schmidt (2009) a prevalência do diabetes vem crescendo mundialmente, configurando-se atualmente como uma epidemia resultante, em grande parte, do envelhecimento da população, tornando-se assim um grande problema de saúde pública no Brasil e no mundo.

Além do envelhecimento populacional, outros fatores importantes têm contribuído significativamente para o aumento do diabetes no mundo, tais como a urbanização acelerada, hábitos de vida inadequada, etilismo, tabagismo, excesso de peso e dieta não controlada (BRASIL, 2006).

A Organização Mundial de Saúde (OMS) estima que o número total de pessoas com diabetes no mundo elevar-se-á, de 171 milhões em 2000 para 366 milhões em 2030; apenas no Brasil, de 4,5 milhões para 11,3 milhões, no mesmo período, tornando-se o oitavo país no mundo com o maior número de pessoas com diabetes (FREITAS; GARCIA, 2012; RATHMANN; GIANI, 2004).

O Diabetes mellitus pode ser definido como um conjunto de alterações metabólicas caracterizadas por hiperglicemia crônica, em decorrência da destruição das células beta do pâncreas, resistência à ação e/ou distúrbios da secreção da insulina (TAVARES, 2011).

O Diabetes mellitus não é uma única doença, mas um grupo heterogêneo de distúrbios metabólicos que apresentam em comum a hiperglicemia (SBD, 2006).

De acordo com Gross et al. (2002) a hiperglicemia se manifesta por sintomas como poliúria, polidipsia, perda de peso, polifagia e visão turva ou por complicações agudas que podem levar a risco de vida: a cetoacidose diabética e a síndrome hiperosmolar hiperglicêmica não cetótica. Os sinais e sintomas evoluem lentamente, dificultando a descoberta e o estabelecimento de diagnóstico precoce. Assim, comumente muitos indivíduos recebem o diagnóstico após a manifestação de complicações da doença (TAVARES, 2011).

Segundo Xavier, Bittar e Ataíde (2009) o Diabetes mellitus é uma doença crônica, em que grande parte de suas complicações torna o indivíduo incapaz de realizar suas atividades cotidianas, o que podecontribuir para uma diminuição de sua autoestima e, consequentemente, afetar sua qualidade de vida. Na prática assistencial, deparamo-nos com o impacto que o Diabetes mellitustem sobre a saúde da população e com a dificuldade de adesão pelos portadores aos comportamentos preventivos.

De acordo com Gross et al. (2002), é extremamente importante atuar na prevenção e no diagnóstico certo e precoce, possibilitando formas de cuidado integral ao paciente com Diabetes mellitus, podendo evitar complicações decorrentes da patologia.

Uma pesquisa realizada por Brasil (2012) em 26 capitais e no Distrito Federal divulgou que no sexo masculino o percentual subiu de 4,4\%, em 2006 para 5,2\% em 2011, entretanto, este aumento da prevalência de homens que informam ter a doença continua sendo inferior ao das mulheres (6\%). Em relação à mortalidade por Diabetes mellitus, um estudo realizado por Belfort; Oliveira (2001) observou que nas mulheres há um percentual maior de óbitos por Diabetes mellitus a partir dos 40-49 anos acentuando-se entre 70-79 anos. A diferença entre os grupos praticamente se igualou por volta dos 80 anos.

De acordo com Aquino (2006), a demanda masculina por serviços ambulatoriais é descrita, em sua maior parte, como gerada pelo trabalho ou pelo seguro social enquanto que a demanda feminina apresenta-se como essencialmente voluntária, revelando uma maior propensão das mulheres a buscarem cuidados de saúde de modo espontâneo, pois as diferenças de gênero na construção da experiência de adoecimento tanto na percepção, quanto no relato diferenciado entre os sexos - também contribuiriam para a existência desses diferenciais de morbidade e de utilização de serviços de saúde.

Esta revisão justifica-se por ser o primeiro passo para que cada vez mais possa despertar nos profissionais de saúde a necessidade de se trabalhar questões de gênero em saúde abrangendo as doenças crônicas não transmissíveis para que os pacientes, tanto homem como mulher, alcancem o melhor estado de saúde biopsicossocial com uma assistência adequada e preventiva respeitando essas diferenças biológicas.

Neste contexto, o presente estudo é resultado de uma revisão de literatura, cujo objetivo deste foi conhecer a prevalência do Diabetes mellitus segundo sexo e a relação com a busca pelos serviços de saúde por homens e mulheres portadores de Diabetes mellitus a fim de sensibilizar os profissionais de saúde o quanto é importante estabelecer programas de atenção tanto a saúde do homem como da mulher portador ou não do Diabetes mellitus. 


\section{METODOLOGIA}

A metodologia empregada para a realização do estudo foi revisão de literatura com coleta de dados secundários em livros, teses, dissertações e artigos disponibilizados na Scientific Electronic Library Online (SCIELO) e no Google Acadêmico.

Para a localização das publicações que compõem o estudo elaborou-se uma estratégia de busca específica nas SCIELO e no Google Acadêmico utilizando-se os seguintes descritores cruzados: Diabetes mellitus, idoso, envelhecimento, cuidados de saúde, educação e saúde.

Para a realização do estudo somente foi selecionado material publicado no período compreendido entre os anos de 2000 e 2011, no idioma português, disponibilizado na íntegra e de forma gratuita, por intermédio da internet.

Após obtenção e seleção prévia das publicações de modo online as mesmas foram submetidas à leitura e as informações relacionadas à temática proposta foram coletadas, organizadas e discutidas.

\section{DESENVOLVIMENTO}

O Diabetes mellitus está diretamente relacionado com a deficiência na produção de insulina, devido à falta desta ou incapacidade de exercer sua função com sucesso (FAEDA; LEON, 2006). A insulina é um hormônio de eficiente ação frente às incapacidades do pâncreas; ela é necessária para reverter o estado catabólico, prevenir a cetoacidose, reduzir o excesso de glucagon e da glicose sanguínea (SOUZA; ZANETTI, 2000).

Segundo Teixeira (2011), o Diabetes mellitus tem sido considerado como um importante problema de saúde na atualidade, tanto em prevalência, incidência e mortalidade prematura, como pelos custos envolvidos no controle e no tratamento de suas complicações. A melhor compreensão das causas e meios que levam às complicações do Diabetes mellitus tem propiciado um tratamento mais adequado e, consequentemente, o aumento da expectativa de vida e maior sobrevida destas. Além disso, a complexidade do tratamento do diabetes no cotidiano, que visa alcançar níveis normais de glicose sanguínea, evitando complicações agudas e buscando uma adequação satisfatória ao estilo de vida, exige que a equipe de saúde multiprofissional esteja capacitada para o atendimento, onde a enfermagem busca contemplar uma abordagem integrada a todos os sistemas corporais.

De acordo com a Organização Pan-Americana de Saúde(OPAS, 2008), existem no Brasil aproximadamente 600.000 portadores de Diabetes mellitus tipo I e cerca de 7.290 .748 de portadores de Diabetes mellitus tipo II. O grande número de portadores de diabetes e a gravidade de suas complicações exprimem porque esta doença é um grande problema de saúde pública, que acarreta um grande ônus para o país, além dos custos inatingíveis como perda da qualidade de vida, ansiedade, inconveniências e outras limitações que provocam grande impacto nos indivíduos portadores e em seus familiares.

O Diabetes mellitus tipo 1 (DM1) representa 5\%-10\% dos casos, estando relacionado à destruição das células beta pancreáticas, tendo como um outro efeito secundário a deficiência de insulina, já o Diabetes mellitus tipo 2 (DM2) está presente em $90 \%-95 \%$ dos casos, é definido pelo mal funcionamento da insulina (SBD, 2006). Atualmente são adotados três critérios aceitos para o diagnóstico de Diabetes mellitus.

A Tabela 1 mostra de forma sintetizada os critérios clínicos e laboratoriais para o diagnóstico de diabetes:

Sintomas de Diabetes mellitus(poliúria, polidipsia, polifagia ou perda de peso inexplicada)

$+$

Glicemia casual: $>\mathbf{2 0 0 m g / d L}$ (realizada a qualquer hora do dia, independentemente do horário das refeições);

$=\mathrm{ou}=$

Glicemia de jejum:> 126mg/dL*;

$=\mathrm{ou}=$

Glicemia de 2 horas: $>200 \mathrm{mg} / \mathrm{dL}$ no teste de tolerância à glicose*.

*Devem ser confirmados com nova glicemia

Tabela 1. Critérios laboratoriais para o diagnóstico de diabetes.

Fonte: Brasil (2006).

Persp. online: biol. \& saúde, Campos dos Goytacazes, 16 (5). P.1-10, 2015

seer.perspectivasonline.com.br 
O Diabetes mellitus não tem cura, entretanto, conforme Faeda e Leon (2006), para o DM2 pode ser oferecido um tratamento com base em dieta nutricional, exercício físico, medicamentos hipoglicemiantes orais e insulina. O foco principal do tratamento é controlar a taxa de açúcar no sangue, podendo ser realizada primeiramente de maneira não medicamentosa, com adoção de hábitos de vida saudáveis e posteriormente com as farmacológicas.

Conforme a Sociedade Brasileira de Diabetes (SBD, 2006), a farmacoterapia deve ser iniciada quando as providências não-farmacológicas forem insuficientes.

Por exemplo, mudanças de estilo de vida reduziram 58\% da incidência de diabetes em 3 anos. Essas mudanças visavam discreta redução de peso (5-10\% do peso), manutenção do peso perdido, aumento da ingestão de fibras, restrição energética moderada, restrição de gorduras, especialmente as saturadas, e aumento de atividade física regular.

Nas intervenções farmacológicas, alguns medicamentos utilizados no tratamento do diabetes, como a metformina, também foram eficazes, reduzindo em $31 \%$ a incidência de diabetes em 3 anos. Esse efeito foi mais acentuado em pacientes com índice de massa corporal(IMC) $>35 \mathrm{~kg} / \mathrm{m}^{2}$ (BRASIL, 2006).

Ao enfermeiro cabe identificar precocemente as complicações que acometem o indivíduo, onde deve atuar intervindo o mais rápido possível. O papel primordial é trabalhar na prevenção evitando que a patologia e as complicações incidam no indivíduo realizando por meio da educação em saúde com enfoque no autocuidado. Além disso, é muito importante que o enfermeiro preste assistência familiar já que os membros desta podem contribuir para o cuidado do paciente com doenças crônicas e, dessa maneira, prevenir ou diminuir as complicações dela decorrentes (PACE; NUNES; VIGO, 2003).

De acordo com Ortiz e Zanetti (2001), o diabetes mellitus é considerado uma das principais doenças crônicas que afetam o homem contemporâneo, acometendo populações de países em todos os estágios de desenvolvimento econômico e social, além de que nas últimas décadas as incidências vêm crescendo em decorrência de vários fatores, como: maior taxa de urbanização, aumento da expectativa de vida, industrialização, maior consumo de dietas hipercalóricas e ricas em carboidratos de absorção rápida, deslocamento da população para zonas urbanas, mudança de estilos de vida tradicionais para modernos, inatividade física e obesidade, sendo também necessário considerar a maior sobrevida da pessoa diabética.

Em estudo realizado por Grillo eGorini (2007) com a finalidade de caracterizar pessoas com DM2, cadastrados em uma Unidade Básica de Saúde (UBS), obteve-se a maior prevalência de pessoas do sexo feminino $84(67,2 \%)$ do que no sexo masculino $41(32,8 \%)$.

Souza (2003) estudou a prevalência do Diabetes mellitus de acordo com a idade e sexo, obtendo como resultado em mulheres após os 40 anos de vida; esta constatação pode estar relacionada ao período de mudanças nas taxas hormonais presente no climatério, o que pode propiciar o aumento da prevalência do Diabetes mellitus neste período da vida.

Ferreira e Ferreiroa (2009) afirma que entre os pacientes diabéticos cadastrados no programa de acompanhamento de Hipertensos e Diabético (HIPERDIA) a predominância pertence ao sexo feminino.

A maior prevalência de Diabetes mellitusem mulheres pode estar relacionada à maior procura dos serviços de saúde (GOLDENBERG;SCHENKMAN; FRANCO, 2003).

Segundo Gomes, Nascimento eAraújo (2007), a procura de serviços de saúde está ligada ao que a cultura da sociedade entende por ser homem ou mulher, ou seja, o papel de cuidadosa está atrelado ao sexo feminino. A mulher tem um maior cuidado para si e para seus filhos, já o imaginário social masculino acredita que não foi criada para se cuidar e sim para trazer renda a toda a família. Tal reflexão traz à tona como os paradigmas e estereótipos estão presentes na sociedade, apontando as diferenças de papéis por gênero, caracterizando erroneamente o cuidado somente como propriedade do sexo feminino.

Outros estudos nacionais e regionais realizados por Malerbi e Franco (1992) constataram que a prevalência de Diabetes mellitus em homens (7,5\%) e mulheres (7,6\%) apresentaram taxas semelhantes, corroborando com o estudo de Torquato et al. (2003), realizado em Ribeirão Preto - São Paulo (SP) que mostrou que mulheres e homens apresentaram taxas semelhantes de diabetes mellitus com índices de 12,1\% e $12 \%$, respectivamente, apontado que não há diferença significativa na prevalência de Diabetes mellitus em relação ao sexo no Brasil.

Sabe-se que o aumento da incidência desta patologia está relacionado há presença de fatores de risco e principalmente ao aumento da idade, este dado é confirmado por Faeda e Leon (2006) mencionando que "em relação ao DM2, atinge indivíduos de qualquer idade, maiores de 40 anos, compreendendo cerca de $7,6 \%$ do total da população brasileira". 
A questão de gênero faz-se pensar o modo que o homem e a mulher pensam sobre sua saúde, sua posição na sociedade e como se viver saudável sendo portador de Diabetes mellitus.

Torrão-Filho (2005) traz que o gênero não se restringe mais a divisão binária do ser humano a partir das construções baseadas sobre o sexo, e nas diferenças percebidas entre os sexos, mas sim de uma forma mais heterogênea, que propõe questionamento das relações sociais.

Scott (1990) argumenta que o conceito de gênero foi criado para opor-se a um determinismo biológico nas relações entre os sexos, dando-lhes um caráter fundamentalmente social. "O gênero enfatizava igualmente o aspecto relacional das definições normativas da feminidade" (SCOTT, 1990, p.5). Este aspecto relacional vem da preocupação de alguns de que os estudos femininos se centravam sobre as mulheres de maneira demasiado estreita, assim, a noção de gênero daria conta de que as mulheres e os homens eram definidos em termos recíprocos e não poderiam ser entendidos separadamente.

Gênero e sexualidade representam as dimensões da vida que são construídas socialmente, sendo que as relações que envolvem as discussões de gênero referem-se à elaboração cultural das identidades feminina e masculina, do ser mulher e do ser homem, observando-se que a sexualidade é uma expressão da personalidade. Associadas ao interior, escuro, fechado e privado, a mulher aprende que do gênero feminino a sociedade espera o ser mãe, o cuidar e o maternar, a dependência, o ser companheira do homem, a pureza, a docilidade (FAGUNDES, 2005).

Segundo Fagundes (2005), o homem, associado ao exterior, claro, aberto e público, aprende que a sociedade espera do gênero masculino a virilidade, a racionalidade, a força, o controle, o engrandecimento de seu trabalho, sua profissão, a produção, sucessos, aventuras, conquistas, o ser provedor da vida e do destino da família, além do controle das emoções, tidas como sinônimo de fragilidade.

A imposição do modelo heterossexual atrelado à subserviência feminina é um fator de destaque na entrada da fase adulta. O homem é ensinado a ser líder, macho e viril, tendo a mulher como submissa e frágil. A questão da homossexualidade é obscurecida e posta de lado,principalmente por ser vista como uma afronta à masculinidade, que não deve ser questionada (VIANA, 2006).

Segundo Figueiredo (2005), a pouca presença do homem nos serviços de atenção primária à saúde leva a muitas suposições e/ou justificativas afirmando que, por um lado, associa-se a ausência dos homens ou sua invisibilidade nesses serviços a uma característica da identidade masculina relacionada a seu processo de socialização. Nesse caso, a identidade masculina estaria associada à desvalorização do auto-cuidado e à preocupação incipiente com a saúde.

Pode ser bem observado em pesquisa por Gomes, Nascimento e Araújo (2007) em que traz a dificuldade que os homens encontram em ir até o serviço de saúde por falta de tempo. Em pesquisa, os autores revelam que o horário de funcionamento dos serviços de saúde não atende às demandas dos homens, por coincidir com a carga horária de trabalho.

Keijzer (2003) observa que o campo da saúde reprodutiva é um dos que mais vem advogando a participação do homem, seja no sentido exclusivamente de informação e como apoio à saúde das mulheres, seja no sentido de reconhecer o homem como sujeito que tem necessidades, assim como direitos sexuais e reprodutivos.

O sexo feminino é contemplado com inúmeros programas do Ministério da Saúde de atenção à saúde da mulher como o Programa de Assistência Integral à Saúde da Mulher (PAISM), fundado no ano de 1984, o mais abrangente e amplo, pois incorporou como princípios e diretrizes as propostas de descentralização, hierarquização e regionalização dos serviços, bem como a integralidade e a eqüidade da atenção. Segundo Brasil (2008), este programa inclui ações educativas, preventivas, de diagnóstico, tratamento e recuperação, englobando a assistência à mulher em clínica ginecológica, no pré-natal, parto e puerpério, no climatério, em planejamento familiar, Doenças Sexualmente Transmissíveis, câncer de colo de útero e de mama, além de outras necessidades identificadas a partir do perfil populacional das mulheres.

Em 28 de maio de 2004, o Ministro da Saúde, Humberto Costa, lançou a Política Nacional de Atenção Integral à Saúde da Mulher: Princípios e Diretrizes, construídos a partir da proposição do Sistema Único de Saúde (SUS), respeitando as características da nova política de saúde. Além de programas específicos da mulher, tem as leis como a Lei Maria da Penha - Lei n. ${ }^{\circ} 11.340$ (BRASIL, 2006) que previne e coíbe a violência doméstica e familiar contra a mulher constituindo tal ato como violação aos direito humanos. 
"O homem associa ir ao médico como uma diminuição no papel masculino, não busca uma atenção primária em saúde que automaticamente seria sua porta de entrada na busca de umaatenção básica. No entanto, os homens são mais propensos a ter o Diabetes mellitus por não fazer nenhum tipo de busca, assim aumentando o risco para complicações, e buscando somente em casos extremos, de dor, por exemplo" (BRASIL, 2006, p.1).

Como afirma Costa-Junior e Maia (2009), os valores próprios da cultura masculina refletem entre os homens maior noção de invulnerabilidade, levando esses indivíduos a emitirem comportamentos de risco e poucas práticas preventivas. Consideram ainda que o padrão masculino seja um indício de pouca procura por assistência médica e por cuidados preventivos em relação à saúde. Relembrando que há escassos programas preventivos de saúde dirigidos aos homens. Incluir a participação do homem nas ações de saúde é, no mínimo, um desafio, por diferentes razões. Uma delas se refere ao fato de, em geral, o cuidar de si e a valorização do corpo no sentido da saúde, também no que se refere ao cuidar dos outros, não serem questões colocadas na socialização dos homens. Por outro lado, alguns quando promovem o cuidado de seu corpo apresentam um extremo fisiculturismo, quando o cuidado de si transforma-se em risco de adoecimentos (KEIJZER, 2003).

Gomes (2003) e Keijzer (2003) advertem que a reivindicação de uma política de saúde mais integral voltada para a população masculina poderia, à primeira vista, ser tomada como um movimento contrário ao empenho de promover programas de gênero destinados à saúde da mulher. No entanto, é a partir da própria dimensão de gênero que se advoga uma abordagem também do masculino, uma vez que tanto homens quanto mulheres necessitam ser vistos em sua singularidade e em sua diversidade no âmbito das relações que estabelecem (GOMES, 2003).

$\mathrm{O}$ conhecimento das singularidades permite melhor aproximação da forma imediatamente relacional do par masculino-feminino, assegurando uma maior densidade de conhecimento das especificidades de cada pólo em interação. Tomando-se em particular as questões da prevenção e da promoção, pode-se perceber que os efeitos do movimento de incluir o homem no debate sobre saúde não se restringem à saúde masculina. Por conseqüência, consegue ganhos para a saúde feminina em temas que só avançam na medida em que se consegue a participação masculina em seu enfretamento (KEIJZER, 2003).

Hardy e Jiménez (2000) consideram que estudos demográficos, que antes só entrevistavam mulheres, começaram a incluir os homens como informantes. Os homens também vêm sendo convidados a participar na atenção à gravidez e ao parto, não sendo considerados responsáveis somente por provocar a gestação. Fato este que nos leva a pensar grandes motivos que impedemo homem ter um acesso mais freqüente ao serviço de saúde, e uma forma de atendimento primário mais eficaz para oferecer a população em geral, visto que o mesmo não tem disponibilidade de horário em que é fornecido tal atendimento ao paciente, coincidindo com o horário de trabalho do homem. O homem deixa visível o preconceito e machismo quando se diz respeito a sua saúde, evidenciando um comodismo do mesmo.

Os programas preventivos oferecidos pela área da saúde estão voltados mais para as mulheres, esquecendo assim que o homem também sente dor, também necessita de uma atenção primária, para ter mais conhecimento sobre o Diabetes mellituse poder identificar os futuros sintomas dessa patologia. De acordo com a Agência de Notícias do Paraná (ANP, 2012) em28 de março de 2012, o Governo do Estado do Parana instituiu através da Lei $n^{\circ}$. 17.099/2012, o Agosto Azul, mês dedicado a fortalecer as ações de prevenção e promoção à Saúde do Homem. O objetivo é fomentar uma mudança cultural, fazendo com que o homem tenha menos resistência em procurar um médico e passe a verificar com mais frequência suas condições de saúde. Durante o todo o mês de agosto, o poder público e a sociedade civil vão promover atividades como audiência pública, debates, oficinas, feiras de saúde e atividades culturais de conscientização, explica o secretário da Saúde, Michele Caputo Neto.

O papel do enfermeiro é fundamental, reconhecendo a importância dessa patologia, a importância das visitas domiciliares tanto para mulher quanto para o homem, mesmo sendo mais fácil encontrar a mulher em casa que o homem, por isso o enfermeiro precisa ser atento e criativo para fazer reuniões, distribuição de panfletos contendo informações claras e objetivas direcionada para os homens, levando informações até eles, ensinando o homem sobre o Diabetes mellitus, estimulando uma mudança no seu comportamento, promovendo sua independência sua independência no auto cuidado e assim é possível prevenir complicações crônicas e/ou agudas. 
O cuidado vem da mulher, assim, temos que modificar o olhar sobre a saúde principalmente do homem, dessa forma, as diferenças vão ser notadas e percebidas. A maioria das mulheres por ser mais atenciosa já pensa em uma alimentação mais ponderada, equilibrada, levando sua saúde sempre em primeiro lugar.

Nessa perspectiva, o enfermeiro, enquanto profissional de saúde engajado na assistência ao portador de doenças crônicas, neste caso com ênfase no diabetes, deve implantar novas práticas de cuidado capazes de promover a saúde dos diabéticos, já que a adesão ao tratamento e o auto cuidado são pontos frágeis da educação em saúde e que, portanto, merecem ser refletidos profundamente.

Segundo Teixeira (2011) para atingir o melhor resultando tanto na prevenção como na reabilitação do paciente portador de Diabetes mellitus, o enfermeiro precisa avaliar constantemente os cuidados prestados, portanto, este profissional deverá assumir suas atribuições por meio da aplicação do método que é o Processo de Enfermagem que propicia uma assistência qualificada, organizada e sistematizada.Portando, o enfermeiro, além de capacitar sua equipe de auxiliares na execução das atividades, deve realizar as consultas de enfermagem, identificar os fatores de risco e de adesão, possíveis intercorrências no tratamento e encaminhar ao médico quando necessário (FAEDA; LEON, 2006).

\section{CONSIDERAÇÕES FINAIS}

No tratamento do DM2 é essencial que o próprio paciente se conscientize da importância de manter o nível glicêmico controlado e que conheça as formas de prevenção de complicações dessa síndrome para obter um estilo de vida adequado. As ações dos profissionais da equipe de saúde envolvidos no cuidar devem ser de maneira articulada e integrada, tendo um enfoque nas ações educativas para elevar o conhecimento dos pacientes, pois estas contribuem grandemente na obtenção de resultados eficazes com vista à adesão ao tratamento e melhoria na qualidade de vida do portador de Diabetes mellitus.

Ainda existe uma grande lacuna no atendimento aos portadores de doenças crônicas não transmissíveis nas UBS, principalmente no acompanhamento do Diabetes mellitus, que se torna uma patologia complexa pela magnitude da incidência e complicações. Os profissionais de saúde não estão devidamente qualificados e capacitados para atender as particularidades individuais dos pacientes que buscam o serviço de saúde.

A prevalência na maior parte dos estudos analisados se constitui do sexo feminino, estes dados trazem consigo reflexões acerca dos motivos que fazem da mulher a mais acometida pelo diabetes. Vários fatores podem ser relacionados como o papel da mulher atrelada a ser cuidadosa, a instabilidade hormonal no climatério, a mulher buscando mais os serviços de saúde, logo é diagnosticada precocemente, além dos programas de saúde estar mais voltados à saúde da mulher, o que facilita o acesso.

Desta forma, é preciso ampliar o olhar para diferentes condições e etapas da vida do paciente, conhecer melhor sobre o processo de envelhecimento e as diferenças na incidência das doenças em ambos os sexos, criando estratégias diferenciadas para que o homem também venha buscar informações e tratamento na UBS, possibilitando identificar quais são os motivos que interferem nesta busca. Proporcionando sempre uma atenção para que os homens passem a ter mais conhecimentos e adquiram hábitos saudáveis, melhorando a perspectiva de vida.

Promover a saúde na demanda masculina é atividade difícil, visto que o mesmo se encontra na maioria das vezes resistente ao cuidado e ausente em decorrência do trabalho. Neste momento o enfermeiro deve atuar com criatividade e inovação, com um olhar diferenciado em colocar em prática os programas preventivos dirigidos aos homens. Algumas estratégias quepossibilitam atrair a população é unir lazer, saúde e interação, promovendo atividades recreativas como caminhadas, palestras, bingos, jogo de baralhos entre outras atividades.

É preciso estabelecer uma assistência humanizada baseada no princípio norteador da equidade, atendendo cada indivíduo de acordo com suas necessidades biopsicossociais, onde tanto homens e mulheres possam buscar os serviços de saúde de forma voluntária e consciente; porém, para tal ação, é preciso identificar e diferenciar a prevalência e acometimento das patologias que acometem homens e mulheres. 


\section{REFERÊNCIAS}

ANP. Agência de Notícias do Paraná. Paraná lança "Agosto Azul" para promover a saúde do homem. 2012. Disponível em: <http://www.aen.pr.gov.br/modules/noticias/article.php?storyid=70144>. Acesso em: 06 mar. 2015.

AQUINO, E. M. L. Gênero e saúde: perfil e tendências da produção científica no Brasil. Revista Saúde Pública, São Paulo,v. 40, n.1, p.121-132, jan. 2006.

BRASIL. Presidência da republica. Câmara dos deputados. Lei Maria da Penha - Lei 11340/06 | Lei ${ }^{\circ}$ 11.340, de 7 de agosto de 2006. 2006.Disponível em: $<$ http://presrepublica.jusbrasil.com.br/legislacao/95552/lei-maria-da-penha-lei-11340-06>.Acesso em: 06 mar. 2015.

BRASIL. Ministério da Saúde. Secretaria de Atenção à Saúde. Departamento de Atenção Básica. Obesidade. Brasília: Ministério da Saúde, 2006, 108p.

BRASIL. Ministério da Saúde. Programa de Assistência Integral à Saúde da Mulher (PAISM), 2008. Disponível em: <http://portal.saude.gov.br/portal/saude/cidadao/ area.cfm? id_area=152>. Acesso em: 29 jun. 2012.

BRASIL. Portal da Saúde. Pesquisa aponta que diabetes é maior em mulheres. 2012. Disponível em: $<$ http://portalsaude.saude.gov.br/portalsaude/noticia/4957/162/pesquisa- aponta-que-diabetes-e-maior-emmulheres.html>. Acesso em: 20 maio 2012.

BERGORT, R.; OLIVIEIRA, J. E. P. O. Mortalidade por Diabetes Mellitus e Outras Causas no Município do Rio de Janeiro - Diferenças por Sexo e Idade. Arquivos Brasileiros de Endocrinologia e Metabólica, São Paulo, v.45, n.5, p. 460-466, out. 2001.

COSTA -JUNIOR, S. D.; OLINTO, M. T. A.; ASSUNÇÃO, M. C. F.; GIGANTE, D. P.; MACEDO, S.; MENEZES, A. M. B. Prevalência de Diabetes Mellitus em Pelotas, RS: um estudo de base populacional. Revista Saúde Pública, São Paulo, v.40, n. 3, p. 542-545, jun. 2006.

COSTA-JUNIOR, F. M.; MAIA, A. C. B. Concepções de homens hospitalizados sobre a relação entre gênero e saúde. Psicologia: Teoria e Pesquisa, Brasília, v. 25, n.1, p. 55-63, jan. 2009.

FAEDA, A.; LEON, C. G. R. M. P. Assistência de enfermagem a um paciente portador de Diabetes Mellitus. Revista Brasileira de Enfermagem, Brasília, v. 59, n.6, p. 818-821, dez. 2006.

FAGUNDES, T. C. P. C. Sexualidade e gênero: uma abordagem conceitual. In: Ensaios sobre educação, sexualidade e gênero. Salvador: Helvécia, 2005. p.9-20.

FERREIRA, C. L. R. A.; FERREIRA, M. G. Características epidemiológicas de pacientes diabéticos da rede pública de saúde: análise a partir do sistema HiperDia. Arquivos Brasileiros de Endocrinologia \& Metabologia, Botucatu, v. 53, n.1, p.80-86, 2009.

FIGUEIREDO, W. Assistência à saúde dos homens: um desafio para os serviços de atenção primária. Saúde Coletiva, Rio de Janeiro, v.10, n.1, p. 105-109, jan. 2005.

FREITAS, L. R. S.; GARCIA, L. P. Evolução da prevalência do diabetes e deste associado à hipertensão arterial no Brasil: análise da pesquisa nacional por amostra de domicílios, 1998, 2003 e 2008. Epidemiologia e Serviços de Saúde, Brasília, v. 21, n.1, p. 1-5, jan. 2012. 
GOLDENBERG, P.; SCHENKMAN, S.; FRANCO, L. J. Prevalência de diabetes mellitus: diferença de gênero e igualdade entre os sexos. Revista Brasileira de Epidemiologia. São Paulo, v. 6, n.1, p.18-28, jan. 2003.

GOMES, R. Sexualidade masculina e saúde do homem: proposta para uma discussão. Ciência e saúde coletiva, Manguinhos, v.8, n. 3, p. 825-829, jun. 2003.

GOMES, R.; NASCIMENTO, E. F.; ARAUJO, F. C. Por que os homens buscam menos os serviços de saúde do que as mulheres? As explicações de homens com baixa escolaridade e homens com ensino superior. Cadernos de Saúde Pública, Rio de Janeiro, v.23, n.3, p.565-574, jun. 2007.

GRILLO, M. F. F.; GORINI, M. I. P. C. Caracterização de pessoas com Diabetes Mellitus Tipo 2. Revista Brasileira de Enfermagem, Brasília,v.60, n.1, p.49-54, jan. 2007.

GROSS, J. L.; SILVÉRIO, S. P.; CAMARGO, J. L.; REICHELT, A. J.; AZEVEDO, M. J. Diabetes Melito: diagnóstico, classificação e avaliação do controle glicêmico. Arquivos Brasileiros de Endocrinologia \& Metabologia, Botucatu, v. 46, n. 1, p.16-26, jan. 2002.

HARDY, E.; JIMÉNEZ, A. L. Masculinidad y género. In: R. BRICEÑO-LEÓN, M. C. S. Minayo, Coimbra Junior C.E. A. (Coord.). Salud y equidad: una mirada desde las ciencias sociales. Rio de Janeiro: Fiocruz, 2000. p.349-367.

KEIJZER, B. Hasta donde el cuerpo aguante: género, cuerpo y salud masculina. In: Cáceres, M.C. (Coord.). La salud como derecho ciudadano: perspectivas y propuestas desde América Latina. Lima: Facultad de Salud Pública y Administración de la Universidad Peruana Cayetano Herida, 2003. p.137-152.

KNOBEL, M. Orientação familiar. Campinas: Papirus, 1992. 56p.

MALERBI, D. A.; FRANCO, L. J. Estudo multicêntrico da prevalência de diabetes mellitus e intolerância a glicose na população urbana brasileira com idade entre 30-69 anos. O Grupo Cooperativo Brasileiro sobre o estudo da prevalência da diabetes. Diabetes Care, Alexandria, v.15, n.11, p.1509-1156, set. 1992.

OPAS. Organização Pan-Americana de Saúde. Diabetes Mellitus. 2008. Disponível em: <www.opas.org.br>. Acesso em: 02 ago. 2012.

ORTIZ, M. C. A.; ZANETTI, M. L. Levantamento dos fatores de risco para diabetes mellitus tipo 2 em uma instituição de ensino superior. Revista Latino-Americana de Enfermagem, Rio de Janeiro, v. 9, n. 3, p.58-63, mai. 2001.

PACE, A. E.; NUNES, P. D.; VIGO, K. O. O conhecimento dos familiares acerca da problemática do portador de diabetes mellitus. Revista Latino Americana de enfermagem, Rio de Janeiro, v. 11, n.3, p.312319, jun. 2003.

RATHMANN, W.; GIANI, G. Global prevalence of diabetes: estimates for the year 2000 and projections for 2030. Diabetes Care, Alexandria, v.27, n.10, p.1047-1053, out. 2004.

SCHMIDT, M. I.; Duncan, B. B.; Hoffmann, J. F.; Moura, L.; Malta, D. C.; Carvalho, R. M. S. V. Prevalência de diabetes e hipertensão no Brasil baseada em inquérito de morbidade auto-referida, Brasil, 2006. Revista de Saúde Pública, São Paulo, v.43, n.2, p.74-82, mar. 2009.

SCOTT, J. W. Gênero: uma categoria útil de análise histórica. Educação e Realidade, Porto Alegre, v. 16, n. 2, p. 1-5, jun. 1990. 
SBD. Sociedade Brasileira de Diabetes. Diretrizes da Sociedade Brasileira de Diabetes. Tratamento e acompanhamento do Diabetes mellitus. 2006. Disponível em: < http://www.diabetes.org.br/educacao/docs/diretrizes.pdf >. Acesso em: 17 mar. 2012.

SOUZA, L. J.; CHAlitA, F. E. B.; REIS, A. F. F. R.; TEIXEIRA, C. L.; GICOVATE- NETO, C.; BASTOS, D. A. et al. Prevalência de diabetes mellitus e fatores de risco in Campos dos Goytacazes, RJ. Arq Arquivos Brasileiros de Endocrinologia \& Metabologia, Botucatu, v. 47, n. 1, p. 69-74, jan. 2003.

SOUZA, C. R.; ZANETTI, M. L. Administração de. insulina: uma abordagem fundamental na educação em diabetes. Revista Escola de enfermagem da USP, São Paulo, v.34, n.3, p.264-70, set. 2000.

TAVARES, B. C.; BARRETO, F. A.; LODETTI, M. L.; SilVA, D. M. G. V.; SILVA, D. M. G. V.; LESSMANN, J. C.Resiliência de pessoas com Diabetes Mellitus. Texto\& Contexto Enfermagem, Florianópolis, v.20, n.4, p.751-757, out. 2011.

TEIXEIRA, C. R. S.; BECKER, T. A. C.; CITRO, R.; ZANETTI, M. L.; LANDIM, C. A. P. Validação de intervenções de enfermagem em pessoas com diabetes mellitus. Revista Escola de enfermagem da USP, São Paulo, v. 45, n.1, p.173-179,jan. 2011.

TORRÃO-FILHO, A. Uma questão de gênero: onde o masculino e o feminino se cruzam. Cadernos Pagu, São Paulo, v. 24, p. 127-152, jan-jun. 2005.

TORQUATO, M. T. C. G.; MONTENEGRO-JUNIOR, R. M.; VIANA, L. A. L.; SOUZA, R. A. H. G.; LANNA, C. M. M.; BIDURIN, C.; FOSS, M. C. Prevalence of diabetes mellitus and impaired glucose tolerance in the urban population aged 30-69 year in Ribeirão Preto-SP, Brazil. São Paulo Medicine Journal, São Paulo, v. 121, n. 6, p. 224-230, 2003.

VIANA, F. O armário: vida e pensamento do desejo proibido. São Paulo: Produção Independente, 2006. $100 \mathrm{p}$.

XAVIER, A. T. F.; BITTAR, D. B.; ATAIDE, M. B. C. Crenças no autocuidado em diabetes: implicações para a prática. Texto \& Contexto Enfermagem, Florianópolis, v. 18, n. 1, p. 124-130, jan. 2009. 